- 140001245

(a) pia

Time bounded computations over the reals

Felipe Cucker José Luis Montaña

Luis Miguel Pardo

Report LSI-91-10

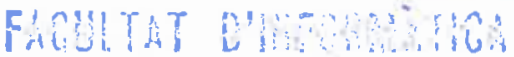

$$
\begin{aligned}
& \text { EISLIOTECA } \\
& \text { R. } 8624 \quad 8 \text { ABR. } 1991
\end{aligned}
$$




\section{Time Bounded Computations over the reals}

\author{
F. Cucker t,t \\ Dept. L.S.I. \\ Facultat d'Informàtica \\ Barcelona 08028 \\ SPAIN
}

\author{
J.L. Montaña ‡ \\ Dept. Matem. Eotad. y C.C. \\ Univeroidad de Cantabria \\ Santander 99071 \\ SPAIN
}

\author{
L.M. Pardo \\ Dept. Matem. Estad. y C.C. \\ Universidad de Cantabria \\ Santander 39071 \\ SPAIN
}

\section{Introduction}

In their 1989 paper [3], L. Blum, M. Shub and S. Smale introduced a model of computation and a theory of recursiveness that accepted an ordered field or ring as alphabet for the space of admissible inputs. A special emphasis was made in the field of the real numbers, $\mathbf{R}$. That work also made an attempt, under the structural approach to complerity, at a classification of the procedures developed in numerical analysis and computational geometry involving real numbers a inputs. In particular, analogues of the $P$ and $N P$ classes were introduced there.

At first glance many similarities can be seen between the new complexity classes, with their relationships, and their Boolean counterparts, a very remarkable one being the existence of complete problems in $N P$ ([3]) and in $P([7])$. However, many basic differences can also be found. Among those differences one stands out: to obtain complexity clases bounding the used space is irrelevant. In [10] it is shown in fact that any recursive set can be decided in linear space.

Another outstanding difference is given by the fact that there are recursive problems without running time bounds for any machine that solves them. A typical example is given by $\mathbb{Z} \subset \mathbf{R}$. In this paper we shall be mainly concerned with the class of problems that can be decided within bounded time. So, we introduce the clase $T B$ of subsets of $R^{\infty}$ recognised by real Turing machines whose running time in the input gize is a well defined function.

In section 1 we study first properties of such a class. In particular, we characterise them in a geometric manner. Let us recall that a semialgebraic subset of $R^{*}$ is any subsetsobtained by finitely many unions and interseccions of sets defined by polynomial inequalities. In [3] it is shown that any recursively enumerable set can be written as a countable union of semialgebraic sets. Shortly afterwards, C. Michaux characterised the recursively enumerable sets, as those subsets of $\mathbf{R}^{\infty}$ that can be written as a countable union of semialgebraic sets defined over a finite extension of $Q$. We extend this line of work by characterising the sets in $T B$. In fact, we show that a met is in $T B$ if and only if all its subsets of words of aise $n$ are semialgebraic subsets of $\mathbf{R}^{n}$.

This result is used to obtain some consequences regarding the topological structure of recursively enumerable sets as well as of sets in $T B$. More precisely, we endow $\mathbf{R}^{\infty}$ with the sum topology, and we show that all open subsets of $R^{\infty}$ are recursively enumerable, and that for any set $L \subset \mathbf{R}^{\infty}$, if $L$ belongs to $T B$ then also their interior and closure belong to $T B$. We think that this is a step towards S. Smale's idea "to bring the theory of computation into the domain of geometry, analyais and topology".

In section 2 we give time hierarchy theorems for $T B$. A theorem requiring time constructibility is given whose statement is analogous to the well known one for the Boolean case. We give also a theorem not requiring time constructibility, thet uses the particular feature of the real model in which any function with domain and images in the integers is computable. However, we pay the price for this generality by requiring the functions not to be sublinear.

Complerity of algorithms dealing with polynomials over a ring is usually expressed as a function of the degree and the number of variables of the input polynomial. In the Boolean case (where the ring is supposed to be finitely codable) both parameters are related to the sise of the polynomial, the degree being expressed

† Partidly aupported by the ESPRIT BRA Program of the EC under contract no. 3075, project ALCOM.

† Partially supported by DGICyT PB 89/0379. 
as a natural number in base two. This is not the case for machines over the reals, where any natural number takes unitary space. Thus, in [3] section 6, it is shown that for any $d \geq 4$ the $d F E A S$ problem of deciding whether a polynomial of degree $d$ has a real root is NP-complete, but no similar statement can be made without restricting the degree. In fact, the $F E A S$ problem of deciding whether an arbitrary polinomial has a real root is not even in $T B$.

In section 3 we introduce several classes of sets that reflect the complexity of problems like the last one we mentioned. In particular we define the clase $P T B$ of sete decided in peendo time bounded to be the class of sets whose function associating the running time to each input is, as a function from $\mathbf{R}^{\infty}$ to $\mathbf{R}$, time bounded. First properties of this clase are that it is elosed under Boolean operations, it is strictly included between $T B$ and the clase of recursive sets, and has complete problems for reductions in $T B$.

Also, we introduce inside $P T B$ the classes $P^{*}$ and $N P^{*}$ of sets that can be decided in polynomial time on their sises and norms (so now time depends on the input iteelf and not only on its sise) by deterministic and non-deterministic machines respectively. It is then thown that $F A S$ is $N P^{*}$ complete for reductions in $P^{*}$.

In a final remark we explain how to extend $P T B$ into a strict hierarchy between $T B$ and the class of recursive sets, that possesses complete problems in each level.

\section{Time Bounded Computations}

Notations along these pages will follow essentially those introduced in [3]. However we shall omitt the qualification "over the reals" for classes of languages and machines, since no confusion can arise with their Boolean counterpart, which does not appear here.

By $\mathbb{R}^{\infty}$ we shall denote the direct sum $\bigoplus_{i \in \mathbb{N}} \mathbb{R}$, i.e. the set of all sequences of real numbers with only finitely many non-mero coordinates. The sige of an element $x \in \mathbf{R}^{\infty}$ is the non-negative integer $|x|=n \in \mathbf{N}$ such that $x_{n} \neq 0$ is the last non-zero coordinate of $x$. Also, in analogy with the Boolean case, we shall call "language" any set included in $\mathbf{R}^{\infty}$ and the language recognired by a machine $M$ will be denoted by $\mathcal{C}(M)$. Moreover, languages recognired by some machine will be called recurgively enumerable (and denoted r.e. for short) and a language that is r.e. both itself and its complement will be called recuraive. For a language $L \subseteq \mathbf{R}^{\infty}$, we shall denote by $L_{n}$ the set of words in $L$ of aice $n \in \mathbb{N}$, i.e. $L_{n}=\{x \in L|| z \mid=n\}$.

The running time $R_{M}(x)$ of a machine $M$ for $z \in \mathcal{L}(M)$ is the number of nodes in $M$ traversed by the computation of $M$ on $x$ until it reaches the output node.

Using these notations, we can define the halting time function associated to a machine $M$ to be

$$
\begin{aligned}
T_{M} & : \mathbb{N} \\
n & \mapsto \mathbb{N} \cup\{+\infty\} \\
n & \sup \left\{R_{M}(x)|x \in \mathcal{C}(M),| x \mid=n\right\}
\end{aligned}
$$

Definition 1.1. For a total mapping $\bullet: \mathbb{N} \rightarrow \mathbf{N}$ we define the complerity clase $D T I M E_{\mathbf{R}}(\&)$ to be $\left\{L \mid\right.$ there is a machine $M$, such that, $L=\mathcal{L}(M)$ and $\left.T_{M}(n) \in O(s)\right\}$.

Definition 1.2. A machine $M$ is said to be a time bounded computation machine if $T_{M}(n)<\infty$, for all $n \in \mathbf{N}$. We shall say that a language is time bounded recursive ( $L \in T B$ for short) iff $L$ is recognied by a time bounded computation machine.

Remarks 1.3. From the definition above it can be obeerved that the class $T B$ is closed under complementation, finite unions and intersections. The newness of the class $T B$ is a particular feature of the real model. In the Boolean one, $T B$ coincides with the class of recuraive sets, a fact that is not shared in our context. The most straightforward example is the ret of the integers $\mathbb{Z} \subset \mathbf{R}$ which is easily seen to be recuraive, but it is not time bounded (cf. theorem 1.8 bellow).

In [3] section 4, proposition 2, a necessary condition is given for a subset $L \subseteq \mathbb{R}^{\infty}$ to be r.e. Shortly afterwards, C. Michaux in [11] modified that condition to obtain a geometrical characterisation of r.e. sets.

In this section we extend Michsux's characterisation to another, for languages in $T B$. In order to do it we recall some notions from real algebraic geometry . 
Definition 1.4. A semialgebraic set $S$ is a subset of rome finitely dimensional real affine space, $S \subseteq \mathbf{R}^{n}$, for $n<\infty$, such that there is a finite number of polynomials $f_{i j} \in \mathbf{R}\left[X_{1}, \ldots, X_{n}\right]$ and sign conditions $\epsilon_{i j} \in\{>0,=0,<0\}$ such that the following equality holds :

$$
S=\bigcup_{j=1}^{r}\left(\cap_{i=1}^{k}\left\{\left(x_{1}, \ldots, x_{n}\right) \in \mathbf{R}^{n} \mid \operatorname{sign}\left(f_{i j}\left(x_{1}, \ldots, x_{n}\right)\right)=\epsilon_{i j}\right\}\right)
$$

Thus, the class of semialgebraic subsets of $\mathbf{R}^{n}$ is the closure under the Boolean operations of the elass of subsets defined by polynomial inequalities in the variables $X_{1}, \ldots, X_{n}$. If all the polynomials $f_{i j}$ have coefficients in a subfield $H \subset \mathbf{R}$ we shall say that $S$ is defined over $H$.

In [3] it is shown that any r.e. set can be written as a countable union of semialgebraic sets. Shortly later, C. Michanx characterised in [11] those unions of semialgebraic sets that are r.e. Let us recall this characterisation.

Definition 1.5. A language $L$ is said to be finitely generated if there is a finitely generated field extension $Q(S)$ of $Q$ and a countable family of semialgebraic sets defined over $Q(S),\left\{A_{n} \subseteq \mathbb{R}^{i_{n}} \mid i_{n}<\infty\right\}$ such that

$$
L=\bigcup_{n \in \mathbb{N}} A_{n}
$$

This notion captures the geometrical structure of r.e. sets, as the following result stated in [11] shows.

Theorem 1.6. For any language $L, L$ is r.e. iff $L$ is finitely generated.

Our next result gives a characterisation of time bounded sets in the same geometrical manner.

Definition 1.7. A language $L$ is said to be finitely presented if there is a finitely generated field extension $\mathbf{Q}(S)$ of $\mathbf{Q}$, such that for every $n \in \mathbf{N}, L_{n}$ is a semialgebraic subset of $\mathbf{R}^{n}$ defined over $\mathbf{Q}(S)$.

Theorem 1.8. For any language $L, L$ is in $T B$ iff $L$ is finitely presented.

Proof:

$\Leftrightarrow)$ Suppose that $L$ recognired by a time bounded computation machine $M$, and let $T_{M}(n)=\bullet(n)$. Let us consider for each $t \in \mathbb{N}$, the set $I_{\ell}$ of all inputs accepted in time $t$. We know that $I_{t, n}:=\left\{x \in I_{\ell}:|x|=n\right\}$ is a semialgebraic set. Moreover there is a finitely generated field ertension of $Q, Q\left(a_{1}, \ldots, a_{r}\right)$, where $\left\{a_{1}, \ldots, a_{r}\right\}$ is the collection of all constants appearing in the description of $M$, such that for all $n \in N$, $L_{n}$ can be described by a finite number of polynomials in $Q\left(a_{1}, \ldots, a_{r}\right)\left[X_{1}, \ldots, X_{n}\right]$. Thus, we have that

$$
L_{n}=\left(\bigcup_{i=1}^{(n)} I_{t, n}\right)
$$

from where we deduce the statement.

$(\Leftarrow)$ Let us now suppose that $L$ is finitely presented. Then there is a finite extension $Q(S)=Q\left(a_{1}, \ldots, a_{r}\right)$ of $Q$ such that for each $n$ the subset $L_{n}$ can be described be a finite system $\Phi_{n}$ of equalities and inequalities as in the definition 1.4. whose polynomials have coefficients in $Q(S)$. Thus, for each $n, L_{n}$ is described by a family

$$
\left\{\left(f_{i j}^{h}, \epsilon_{i j}^{i}\right)\right\}
$$

where $\epsilon_{i j}^{R}$ is a sign and $f_{i j}^{R} \in Q(S)\left[X_{1}, \ldots, X_{n}\right]$.

Now, each $f_{i j}^{n}$ can be expressed as a polynomial in $Q\left[a_{1}, \ldots, a_{r}, X_{1}, \ldots, X_{n}\right]$ and then can be coded as an element of $N^{\infty}$. Thus, the same happens with the whole family $\left\{\left(f_{i j}^{n}, \epsilon_{i j}^{n}\right)\right\}$. We so consider the function

$$
\begin{aligned}
\varphi: \mathbb{N} & \rightarrow \mathbb{N}^{\infty} \\
n & \rightarrow \text { the encoding of }\left\{\left(f_{i j}^{n}, \epsilon_{i j}^{n}\right)\right\}
\end{aligned}
$$

toghether with the machine given by 


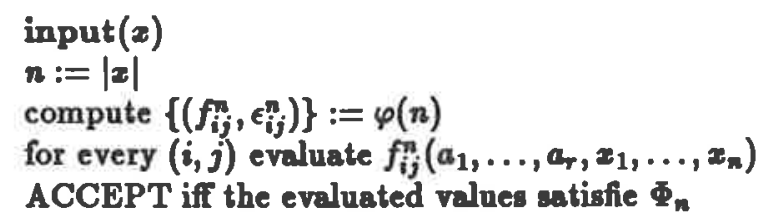

The preceding machine accepts exactly $L$. Moreover, it does so in bounded time. In fact, the first asignement is done in constant time, and the other intructions in time that clearly dependens only on $n$ (and not on the specific input $x$ ).

Remarks 1.0. A non-uniform model of computation frequently used in algebraic complexity to show lower bounds is the algebraic tree (see for instance [1] or [14]). One of the most interesting festures of the model introduced by Blum, Shub and Smale is that an algebraic tree is just a finite acyclic real Turing machine whose computation nodes performe only elementary operations $(t, *,-$ or $/)$. In this sense the computational device introduced in [3] uniformises the algebraic trees. On the other hand, we could also consider as non-uniform models the finite real Turing machines.

The preceding results show that there is a strong relation between computability and geometry, in the sense that there is a correspondence between abstract computational devices and geometrical properties satisfied by the sets recognised by them. We can sumarise them in the following table.

\begin{tabular}{|c|c|}
\hline Machine model & Recognised sets \\
\hline Real Turing Machine & Finitely generated \\
Time Bounded Machine & Finitely presented \\
Finite Machine & Countably unions of semialgebraics \\
Algebraic Tree or & Semialgebraic \\
Algebraic Circuit & . \\
\hline
\end{tabular}

One of the goals stated in [3] with respect to the introduction of the real Turing machine is "to bring the theory of computation into the domain of geometry, analyais and topology". This goal passes trought the fact that $\mathbf{R}$ is endowed with a very rich topological structure.

There is a canonical form to endow a direct sum with a topological structure. The topology induced over $\bigoplus_{i \in N} R$ is called sum topology and their open sete are the sets $U$ such that for every $n$ the set $U_{n}=U \cap \mathbf{R}^{n}-\left\{X_{n}=0\right\}$ is open. In the case of finite sums $\oplus_{1<i<n} \mathbf{R}$, the sum topology coincides with the Euclidean one. For definitions as well as for basic properties of the sum topology, see [5] I, p.15.

\section{Theorem 1.10.}

i) For $k \in \mathbf{N}$, every open subset of $\mathbf{R}^{k}$ is r.e. Thus, a closed subset of $\mathbf{R}^{k}$ is r.e. iff it is recursive.

ii) Assume $\mathbf{R}^{\infty}$ is endowed with the sum topology. Then,

a) Every open set in $\mathbf{R}^{\infty}$ is r.e.

b) A closed subset of $\mathrm{R}^{\infty}$ is r.e. if and only if it is recuraive.

c) For a language $L \subseteq \mathrm{R}^{\infty}$ we denote by $\operatorname{Int}(L)$ and $C \operatorname{los}(L)$ its interior and closure respectively. Then, if $L \in T B$ we also have that $\operatorname{Int}(L), C l o s(L) \in T B$.

Proof: Parts i), ii.e) and ii.b) are consequences of the existence of a good basis of open sets. In fact, any open subset of $R^{n}$ can be written as a countable union of balls $B(x, \epsilon)$ with $x$ has rational coordinates and $\epsilon$ belongs to $Q$. Since these balls are semialgebraic, the statement clearly hold.

For part ii.c) let us consider a set $L \in T B$. For each $n_{2}$ we have a formula $\Phi_{n}\left(x_{1}, \ldots, x_{n}\right)$ defining $L_{n}$, because of the theorem 1.8. Again, because of the structure of the open sets in $R^{\infty}$, it is immediate that

$$
\operatorname{Int}(L):=\bigcup_{n \in \mathbb{N}} \operatorname{Int}\left(L_{n}\right)
$$


and

$$
C \log (L):=\bigcup_{n \in \mathbb{N}} C \operatorname{los}\left(L_{n}\right)
$$

where the interior and closure of $L_{n}$ is taken in $\mathbf{R}^{n}-\left\{x_{n}=0\right\}$. Thus, $\operatorname{Int}\left(L_{n}\right)$ and $C \log \left(L_{n}\right)$ can be expressed by the formula

$$
\exists \epsilon \forall y_{1} \ldots \forall y_{n} \epsilon>0 \wedge \Phi_{n}\left(x_{1}, \ldots, x_{n}\right) \wedge\left[\sum_{k=1}^{n}\left(x_{k}-y_{k}\right)^{2} \geq \epsilon \vee \Phi_{n}\left(y_{1}, \ldots, y_{n}\right)\right]
$$

and

$$
\forall \epsilon \exists y_{1} \ldots \exists y_{n}\left[x_{n} \neq 0 \wedge \epsilon>0 \wedge \Phi_{n}\left(x_{1}, \ldots, x_{n}\right) \wedge \Phi_{n}\left(y_{1}, \ldots, y_{n}\right)\right]
$$

respectively.

These formule only use constants in $Q(S)$. Thus, by the quantifier elimination in the theory of the reals they are equivalent to quantifier-free formulæ with constants in $Q(S)$, which define semialgebraic subsets of $R^{n}-\left\{x_{n}=0\right\}$. The statement is now a trivial consequence of theorem 1.8 .

\section{Time Hierarchy Theorems within TB}

We begin this section with a lemms providing a lower bound for the running time of time bounded machines, that will be useful for proving the hierarchy theorems.

Lemma 2.1. Let $M$ be a time bounded machine and $L$ its accepted language. Then, we have $T_{M}(n) \in$ $\Omega\left(\log _{2} B_{0}\left(L_{n}\right)\right)$, where $B_{0}\left(L_{n}\right)$ denotes the number of connected components of $L_{n}$.

Proof: For every $y \in L_{n}$ we have a sequence of configurations given by the computation of $M$ on $y$

$$
\left(n_{0}, x_{0}\right) \rightarrow\left(n_{1}, x_{1}\right) \rightarrow \cdots \rightarrow\left(n_{k}, x_{k}\right)
$$

where $n_{0}$ is the input node, $n_{k}$ is the output node and $k \leq T_{M}(n)$. By $\gamma(y)$ we shall denote the computation path $n_{0} \rightarrow n_{1} \rightarrow \cdots \rightarrow n_{k}$ in $M$ and let us define

$$
\Gamma_{n}:=\left\{\gamma(y) \mid y \in L_{n}\right\}
$$

the set of all posible paths followed by inputs in $L_{n}$.

Now, $\Gamma_{n}$ is a finite set because the length of every path in $\Gamma_{n}$ is bounded above by $T_{M}(n)$ and the number of path in $M$ of a given length $\leq K$, is bounded by $2^{K}$.

Nert, for every path $\gamma \in \Gamma_{n}$ there is a semialgebraic subset $V_{\gamma} \subseteq \mathbf{R}^{n}-\left\{x_{n}=0\right\}$, given by $V_{\gamma}=\{x \in$ $\left.L_{n} \mid \gamma(x)=\gamma\right\}$, observing that

$$
L_{\pi}=\bigcup_{\gamma \in \Gamma_{*}} V_{\gamma}
$$

At this point let us note that there is a constant $K_{M}$, depending only on the machine $M$, such that for every $n \in \mathbf{N}$ and for every $\gamma \in \Gamma_{n}$, the membership problem to $V_{\gamma}$ can be solved by a Straight Lin e Program $\pi_{\gamma}$ of length $l \leq K_{M} \cdot T_{M}(n)$. At every computation node of $\pi_{\gamma}$ just an arithmetic operation among $\{+,-, x, /\}$ is performed.

Now, we follow the central arguments in [1] plus a little topological trick. Traversing $\pi_{\gamma}$ we get a system of equations $S$ according to the operations or tests on the vertices of $\pi_{\gamma}$. In this system, we add a new variable at each computation node. Let $u_{1}, \cdots, u_{r}$ be the set of variables added in $S$. If $U$ denotes the subset of $R^{n+r}$ defined by $S$ it is clear that the projection on the first $n$ coordinates of $U$ is exactly $V_{\gamma}$.

Thus, the number of connected components of $V_{\gamma}, B_{0}\left(V_{\gamma}\right) \leq B_{0}(\sigma)$. Note that the degree of the system $S$ is bounded above by 2 , the number of equations is bounded by $l$, and the number of active variables $\leq 3 l$, at moat three at each computation node, two due to the computation and one added in $S$ to decrease the degree.

Now, by [1] Theorem 2, it follows that the number of connected components of $V_{\gamma} \leq 2 \times 3^{4-1}$. Since the cardinal of $\Gamma_{n}$ is $\leq 2^{T_{M}(n)}$, the statement follows by observing that

$$
B_{0}\left(L_{n}\right) \leq \sum_{\gamma \in \Gamma_{n}} B_{0}\left(V_{\gamma}\right) \leq 2^{T_{M}(n)+1} \times 3^{4 \cdot K_{M} \cdot T_{M}(n)-1}
$$




\section{Remarks 2.2.}

i) Lemma 2.1 is an improvement of Ben-Or's lower bound in [1], based on the geometry of the semialgebraic sets. In [13] and [12], the lower bounds obtained for the total complerity of a semialgebraic set can also be applied to this model. Moreover, observe that all these lower bounds also hold for the corresponding measure of complerity of machines over any real closed field considered.

ii) The introduction of this lower bound is motivated by the following time hierarchy theorem and its good accuracy for classes of "small" time bound (i.e. lower than linear time) for which the lower bounds in [1] and [12] are not useful at all.

Definition 2.3. A total mapping s: $\mathbf{N} \longrightarrow \mathbf{N}$ is aid to be time constructible if there is a machine $M$ such that the mapping $\varphi_{M}$ computed by $M$ restricted to $\mathbf{N}$ coincides with $s$ and, there is a constant $K \in \mathbf{R}, K>0$ such that $R_{M}(m) \leq K . s(m)$, for all excepting a finite number of elements $m \in \mathbf{N}$, where $R_{M}(m)$ denotes the running time of $M$ on input $m \in \mathbf{N}$.

The most common functions used as time boundo in complexity theory (as, for instance, $\log _{2}^{k}(n), n^{k}$ or $2^{k . n}$, with $k \in \mathbf{N}$ fixed ) are time constructible over the reals.

Theorem 2.4. Let $t, t^{\prime}: \mathbf{N} \longrightarrow \mathbb{N}$ be two time bound, $t^{\prime}$ time constructible and assume that $t^{\prime} \in \omega(t)$. Then, DTIME $E_{\mathbf{R}}\left(t^{\prime}\right)$ contains a language which is not in DTIM $E_{\mathbf{R}}(t)$.

Proof: Let us consider the language $L$ defined as the set of points $x \in \mathbf{R}^{\infty}$, such that $x_{1}+i x_{2}$ is a $2^{t^{\prime}(n)}$ -th root of the unity in the complex plane. The following machine

$$
\begin{aligned}
& \text { input }(x) \\
& n:=|x| \\
& m:=t^{\prime}(n) \\
& z:=\left(x_{1}+i x_{2}\right)^{2^{m}} \\
& \text { if } z=1 \text { then ACCEPT } \\
& \text { fi else REJECT }
\end{aligned}
$$

accepts $L$ in time $O\left(t^{\prime}(n)\right)$. In fact, the first asignement takes constant time, and the following two can be done within time $O\left(t^{\prime}(n)\right)$. But, the number of connected components of $L_{n}$ is $2^{t^{\prime}(n)}$ and so, by lemman 2.1, $L$ cannot be recognised in time $t(n)$.

Remarks 2.5. It is interesting to note that, in contrast with the Boolean case where the input aise is computed in linear time, in the real model, we can compute it in constant time. This fact gives to the real model the capacity of deciding more things in sublinear time.

In the following corollary we present some of the strict inclusions for the most usual complexity classes.

Corollary 2.6. For $k \in \mathbf{N}$ fired, in the following chain the inclusion relations are strict:

i) DTIME $E_{\mathrm{R}}\left(\log _{2}^{k}(n)\right) \subset D T I M E_{\mathrm{R}}\left(\log _{2}^{k+1}(n)\right) \subset D T I M E_{\mathrm{R}}(n)$

ii) DTIME $E_{\mathrm{R}}\left(n^{k}\right) \subset D T I M E_{\mathrm{R}}\left(n^{k+1}\right) \subset D T I M E_{\mathrm{R}}\left(2^{n}\right)$

iii) $D T I M E_{\mathrm{R}}\left(k^{\mathrm{n}}\right) \subset D T I M E_{\mathrm{R}}\left((k+1)^{n}\right) \subset D T I M E_{\mathrm{R}}\left(2^{2^{n}}\right)$.

Lemma 2.7. For every partial mapping $\&: \mathrm{N} \longmapsto \mathrm{N}$ there is a machine $M$ computing s within time $O\left(s(n)(n+s(n))^{2}\right)$ on input $n$.

Proof Let us recall from [3] section 1, example 6, that given a set $S \subset \mathbb{N}$ we can decide whether a real number $x$ belongs to $S$ within time $O(\lfloor x\rfloor)$. Now, we consider the polynomial

$$
f(n, m)=\frac{1+n+m}{2}(n+m)+(m+1)
$$

the set $S=f(\operatorname{graph}(s))$ and the algorithm 


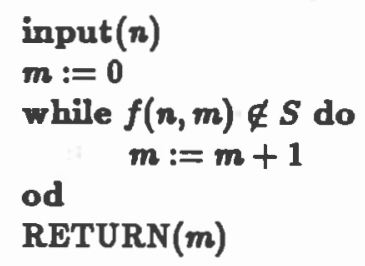

This algorithm computes $\bullet(n)$. Moreover, for a given inpat $n$ we pass $\bullet(n)$ times through the while condition. To check this conditions costs by the preceding remark $O(f(n, m))$ steps. So, the total cost is bounded by $O\left(s(n)(n+s(n))^{2}\right)$.

Remarks 2.8. The preceding result mark another difference with the Boolean model. In this last one, there are many functions not computable from $\mathbf{N}$ to $\mathbf{N}$, bat all time bounds are computable by just getting for each $n$ the maximum of the running times among the $2^{n}$ words of sive $n$. In the real model, this argument obviously does not apply, but the preceding lemma makes it unnecessary in several situations. As an application we can give another time hierarchy theorem for non-neceasarily constructible time bounds .

Theorem 2.8. Let $t, t^{\prime}: \mathbf{N} \rightarrow \mathbf{N}$ be two time bounds, $t^{\prime}(n) \geq n$ and assume that $\sqrt[3]{t^{\prime}} \in w(t)$. Then, $D T I M E_{\mathrm{R}}\left(t^{\prime}\right)$ contains a language not in $D T I M E_{\mathrm{R}}(t)$.

Proof Call $s(n)$ the integer part of the function $\sqrt[3]{t^{\prime}}$ and consider the language $L$ defined by the set of points $\left(x_{1}, \cdots, x_{n}, \cdots\right) \in \mathbb{R}^{\infty}$ such that $x_{1}+i x_{2}$ is a $2^{(n)}$-th root of the unit in C.

A machine $M$ accepting this language within time $O\left(t^{\prime}\right)$ can be designed in the same manner as in the proof of theorem 2.4 substituting $t^{\prime}$ by s. Because of the lemma 2.7 this machine evaluates on the input sise requiring $O\left(t^{\prime}\right)$ steps to complete the evaluation. Next, using $\bullet(|x|)$, the machine controls the simulation on the first two coordinates of the input the complex operation $\left(x_{1}+i . x_{2}\right)^{2(|x|)}$, doing this in $O(s)$ steps. Finally, it compares to $(1,0)$ in constant time, accepting if and only if the equality holds.

The fact that $L$ is not in $D T I M E_{\mathbb{R}}(t)$ follows again in the same manner as in the proof of theorem 2.4 by using lemme 2,1 .

\section{Pseudo time bounded computations over the reals}

Complexity of algorithms dealing with polynomials over a ring is nsually expressed as a function of the degree and the number of variables of the input polynomial. In the Boolean case (where the ring is supposed to be finitely codsble) both parameters are related with the sise of the polynomial, the degree being expreased as a natural number in base two. This is not the case for machines over the reals, where any natural number takes unitary space. Thos, in [3] section 6 , it is shown that for any $d \geq 4$ the problem

$$
d F E A S=\{F \mid F \text { is a polynomial of degree } d \text { that has a real root }\}
$$

is NP-complete, but no similar statement can be made without restricting the degree. In fact, the problem

$$
F E A S=\{F \mid F \text { is a polynomial that has a real root }\}
$$

is not even in $T B$.

In this section we shall introduce several classes of sets lying inside $\boldsymbol{R}$ that reflect the complexity of problems like $F E A S$.

Definition 3.1 Let $L$ be a recursive set. We shall say that $L$ is a Pseudo Time Bonnded set (briefly $L \in P T B$ ) if there is a machine $M$ accepting $L$, and a time bounded recursive function $f: \mathbb{R}^{\infty} \rightarrow \mathbb{N}$ such that $R_{M}=O(f)$.

First properties of $P T B$ can be resumed in the following result. 


\section{Proposition 3.2}

i) The class PTB is closed under complemento and finite unione and intersections.

ii) We have the strict inclusions $T B \subset P T B \subset R$.

iii) The set $K=\{(M, x, t) \mid M$ accepts $x$ in less than $t$ steps $\}$ is PTB-complete for reductions in $T B$.

Proof:

i) Immediate.

ii) Both inclusions being obvious, we shall only show that they are strict.

For the first one, we just note that $\mathbb{Z}$ is not in $T B$ but it is certainly in $P T B$ since there is a machine deciding this set in time $O(x)$ for input $x$, and the identity function is computed in constant (and then bounded) time.

For the second one, let us consider the set

$$
S=\left\{(x, y, t) \mid(x+i y)^{2^{2(1)}}=1\right\}
$$

For a fired value of the parameter $t$ we apply lemma 2.1 getting a lower bound of $2^{t}$ in the number of steps we must perform to decide whether a triple $(x, y, t)$ belongs to $S$. So, any machine deciding $S$ has $2^{t}$ as lower bound for its running time. But any time bounded function with domain $\mathbb{R}^{3}$ must be semialgebraic, and then, must have polynomial growth. We deduce that $S \notin P T B$. On the other hand, $S$ is obviously recursive.

iii) The problem is in PTB since the computing time for an input $(M, x, t)$ is linear in $t$ and then can be obtained in bounded time in the sise of $(M, x, t)$. On the other hand, for any machine $M \in P T B$ we consider the reduction mapping $x(M, x, t)$, where $t$ is obtained from $M$ and $x$ in bounded time since $M \in P T B$.

We shall go now a bit further within $P T B$. In particular, let us recall that for any $x \in \mathbf{R}^{\infty}$ ita norm $\|x\|$ is defined to be $\max \left\{\left|x_{1}\right|, \ldots,\left|x_{|x|}\right|\right\}$. We une this notion to introduce complerity classes within PTB.

Definition 3.3. We shall say that a machine $M$ works in unrestricted polynomial time when for any $x \in \mathcal{L}(M), M$ accepts $x$ within time $O\left((|x| \cdot|| x||)^{k}\right)$ for some $k \in \mathbf{N}$. The class of sets recognired by machines working in unrestricted polynomial time will be denoted by $p u$. In a similar way we define the class of functions computed in unrestricted polynomial time as well as the class $N P^{u}$ of sets accepted in non-deterministic unrestricted polynomial time.

Also, we shall say that a machine $M$ works in unrestricted exponential time when for any $x \in \mathcal{L}(M), M$ accepts $x$ within time $O\left(\left(\|x\|^{k} 2^{|x|}\right)\right.$ for some $k \in \mathbf{N}$. The class of sets recognised by machines working in unrestricted exponential time will be denoted by $E X P^{\mathrm{s}}$.

The main result for these classes is the following

Theorem 3.4. The set FEAS is N $P^{\mathrm{u}}$-complete for reductions in $P^{*}$.

Proof: Clearly FEAS belongs to $N P^{w}$. In order to show the hardness, for any $N P^{u}$ machine $M$ we consider the reduction that to any $x \in \mathbf{R}^{\infty}$

first compute a bound $t=(|x| \cdot|| x||)^{k}$ on the running time of $M$ for $x$

secondly the same reduction given in section 6 of [3] applies, obtaining a system of $t$ equations (in fact, the last part of the quoted reduction is not necessary now because we do not need to decrease the degree).

Proposition 3.5. We have the inclusions $P^{*} \subset N P^{*} \subset E X P^{*} \subset P T B$.

Proof: The only non immediate inclusion is the second one. To prove it we just note that to decide an existencial sentence in the theory of the reals can be done in time bounded by $O\left((k d)^{O(n)}\right)$ where $k$ is the number of polynomials appearing in the sentence and $d$ is a bound on their degree (see [6], [8], [9] or [15] for such algorithms, this last reference making explicit the Blum, Shub and Smale model). Thus, the inclusion follows from the preceding theorem. 
Final remarks s.6.

Research concerning pseudo time bounded computations can be continued following two directions. On the one hand, more complerity classes can be defined inside PTB, and hierarchies as well as well as completeness results can be pursued. On the other hand, a hierarchy of classes over $P T B$ can be inductively defined in the following way. We denote by $P T B_{0}=T B, P T B_{1}=P T B$, and $P T B_{k+1}$ is defined from $P T B_{k}$ in the same manner as $P T B$ was defined from $T B$. A result aimilar to proposition 3.2 can be obtained for each level of this hierarchy.

\section{References.}

[1] M. Ben-Or; "Lower bounds for algebraic computation trees". A.C.M. 15 th Symp. on Theory of Computing, Pp.80-86, 1983.

[2] R. Benedetti and J.J. Rimler; Real algebraic and semialgebraic sets. Hermann, Paris, 1990.

[3] L. Blum, M. Shub and S. Smale; "On a theory of computation and complexity orer the real numbers: NPcompleteness, recursive functions and univeral machines". Bulletin of the Amer. Math. Soc., vol.21, n.1, Pp.1-46, 1989.

[4] J. Bochnak, M. Coate and M.-F. Roy; Géométrie algebrique réelle. Ergebninse der Math., 3.Folge, Band 12, Springer Verlag, 1987.

[5] N. Bourbaki; Éléments de Mathématique. Topologie Général. Chapitrea I-IV. Hermann, Paris, 1971.

[6] J. Canny; "Some algebraic and geometric computations in PSPACE". A.C.M. 20 th Symp. on Theory of Computing, pp.460-467, 1988.

[7] F. Cucker and A. Torrecillas; "Two P-complete problems in the theory of the realo". To appear in Proceedings of ICALP'91, Madrid, 1991.

[8] D. Grigor'ev; "Complexity of deciding Tarubi algebra". J. of Symb. Comp., 5 pp.65-108, 1988.

[9] J. Heints, M.-F. Roy and P. Solerno; "On the complexity of semialgebraic nets". Proceedings of IFIP'89, pp.293298, North Holland, 1989.

[10] C. Michaux; "Une remarque à propos des machines sur $\mathrm{R}$ introduites par Blum, Shub et Smale". C. $R$. Acad. Sci. Paris, t.309, Série I, Pp.435-437, 1989.

[11] C. Michnux; "Machines sur les réels et problèmes NP-complets", Séminaire de Structures Algébriques Ordonnées. Prépublications de l'equipe de logique mathématique de Paris 7, 1990.

[12] J.L. Montaña, L.M. Pardo and T. Recio; "The non-scalar model of complerity in computational geometry". To appear in Proceedings of MEGA' 90 , Birthiuser.

[13] L.M. Pardo and T. Recio; "Rabin's width of a complete proof and the width of a semialgebraic set". Proceedings of the EUROCAL'87, LNCS 378, Springer Verlog, pp.456-462, 1989.

[14] M. Robin; "Proving simultaneou ponitivity of linenr forms". J. of Comp. and Syot. Se., 6, pp.632-650, 1972.

[15] J. Renegar; "On the computational complerity and geometry of the firat order theory of the reala". Technical report 85S, Cornell University, Ithace, 1989. 